\title{
Optical diagnosis of actinic cheilitis by infrared spectroscopy
}

\author{
Luis Felipe das Chagas e Silva de Carvalho (PhD, Postdoctoral researcher) ${ }^{\mathrm{a}, *}$, \\ Thiago Martini Pereira (PhD, Associate Professor) ${ }^{\mathrm{b}}$, \\ Taciana Depra Magrini (PhD, Associate Professor) ${ }^{\mathrm{a}}$, \\ Ana Sueli Rodrigues Cavalcante (PhD, Associate Professor) ${ }^{\mathrm{c}}$, \\ Herculano da Silva Martinho (PhD, Associate Professor) ${ }^{\mathrm{d}}$, \\ Janete Dias Almeida (PhD, Associate Professor $)^{\mathrm{c}}$ \\ a Laboratory of Biomedical Vibrational Spectroscopy, Universidade do Vale do Paraíba, São José dos Campos, São Paulo, Brazil \\ b Instituto de Ciência e Tecnologia, Universidade Federal de São Paulo, São José dos campos, São Paulo, Brazil \\ ${ }^{c}$ Department of Biosciences and Oral Diagnosis, Institute of Science and Technology, Univ Estadual Paulista (UNESP), São José dos Campos, São Paulo, Brazil \\ ${ }^{\mathrm{d}}$ Centro de Ciências Naturais e Humanas, Universidade Federal do ABC, Santo André, São Paulo, Brazil
}

\section{A R T I C L E I N F O}

\section{Article history:}

Received 17 May 2016

Received in revised form 28 June 2016

Accepted 30 July 2016

Available online 1 August 2016

\section{Keywords:}

Actinic cheilitis

Optical biopsy

FT-IR spectroscopy

Oral cancer

\begin{abstract}
A B S T R A C T
Actinic cheilitis (AC) is considered a potentially malignant disorder of the lip. Biomolecular markers study is important to understand malignant transformation into squamous cell carcinoma. Fourier transform infra red (FT-IR) spectroscopy was used to analyze AC in this study.

Objectives: The aim of the study was to evaluate if FT-IR spectral regions of nucleic acids and collagen can help in early diagnosis of malignant transformation.

Methods: Tissues biopsies of 14 patients diagnosed with AC and 14 normal tissues were obtained. FTIR spectra were measured at five different points resulting in 70 spectra of each. Analysis of Principal components analysis (PCA) and linear discrimination analysis (LDA) model were also used. In order to verify the statistical difference in the spectra, Mann-Whitney $U$ test was performed in each variable (wavenumber) with $p$-value $<0.05$.

Results: After the Mann-Whitney $U$ test the vibrational modes of CO (Collagen 1), PO2 (Nucleic Acids) and $\mathrm{C}=\mathrm{O}$ asymmetric (Triglycerides/Lipids) were observed as a possible spectral biomarker. These bands were chosen because they represent the vibrational modes related to collagen and DNA, which are supposed to be changed in AC samples. Based on the PCA-LDA results, the predictive model corresponding to the area under the curve was 0.91 for the fingerprint region and 0.83 for the high wavenumber region, showing the greater accuracy of the test.

Conclusions: FT-IR changes in collagen and nucleic acids could be used as molecular biomarkers for malignant transformation.
\end{abstract}

(C) 2016 Elsevier B.V. All rights reserved.

\section{Introduction}

Recently, new diagnostic methods have been developed aiming to detect molecular changes in the pathological processes in its initial phase. Treatment of late-stage diseases diagnosis is more difficult and expensive. It affects prognosis and survival rates [1,2].

\footnotetext{
* Corresponding author at: Corresponding autor: Laboratory of Biomedical Vibrational Spectroscopy, Avenida Shishima Hifumi, 2911 - Urbanova, São José dos Campos - SP, CEP: 12244-000, Brazil.

E-mail address: luisfelipecarvalho@hotmail.com (L.F. das Chagas e Silva de Carvalho).
}

Actinic cheilitis (AC) is considered a potentially malignant disorder of the lower lip. It has a slow clinical course and shows edematous and atrophic aspect (Fig. 1). In advanced cases, superficial ulcerations and leukoplasic areas can be seen. The lesion is mainly observed in patients over 45 years, light-skinned men working outdoors chronically exposed to sunlight.

$A C$ is caused due to chronic and excessive exposure to ultraviolet (UV) radiation. This condition occurs most frequently in Caucasian patients exposed to ultraviolet (UV) radiation. UV radiation has potential carcinogenic effects.

Histopathological changes in AC are observed in the epithelial and connective tissue. The epithelium is characterized by an atrophic appearance. In some cases, areas of marked acanthosis and 


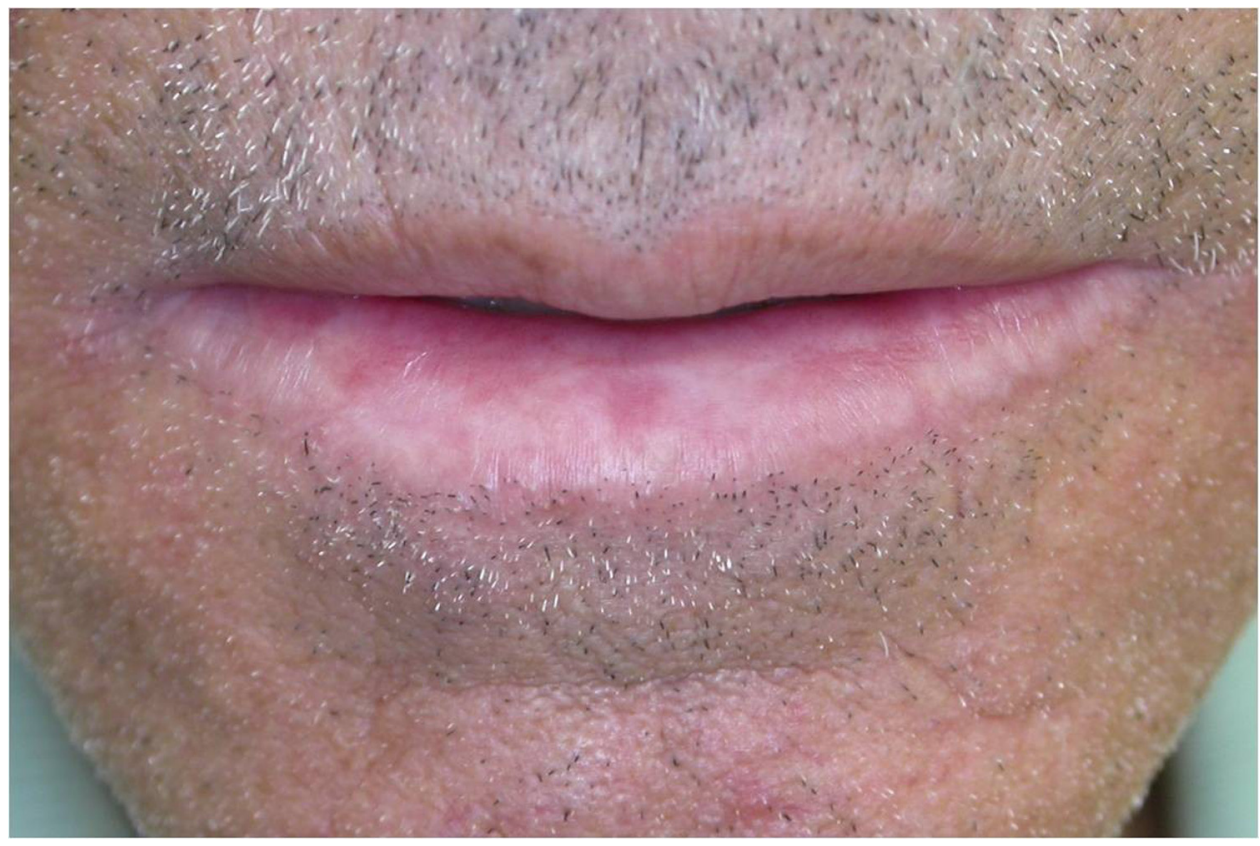

Fig. 1. AC clinical aspect showing multifocal lesions as dryness, pallor, and blurred demarcation between the vermilion border and the skin.

keratin production are observed, which presents variable thickness. Atypia may be observed ranging from mild to severe [3,4]. In the connective tissue, solar elastosis is seen as basophilic degeneration of collagen [3-6].

Actually, biopsies are required for an accurate diagnosis of $\mathrm{AC}$ related to its malignant potential ${ }^{1}$. This procedure has some disadvantages such as affecting the aesthetics and a delay in obtaining a diagnosis due to laboratory procedures. Thus, it is important to develop new techniques for noninvasive diagnostic procedures that can provide the necessary information about the tissue in real time. Also, the study of biomolecular markers is important in terms of AC malignant transformation [3-6].

Non-invasive diagnostic techniques currently studied: Raman spectroscopy, Fluorescence spectroscopy, Infrared spectroscopy (FT-IR). The analysis of ex vivo samples enables the characterization of biological and molecular constituents based on the analysis of chemical structure and conformation after light stimulation [1,7-9].

FT-IR analysis of AC is possible since it may present DNA damage and histological features related to collagen structures changes. The aim of the study was to evaluate if nucleic acids and collagen FT-IR spectral regions can help in early diagnosis of malignant transformation.

Table 1

Correlation between spectral regions and vibrational modes/structural components [27].

\begin{tabular}{lll}
\hline Band $\left(\mathrm{cm}^{-1}\right)$ & Vibrational Mode & Structural Component \\
\hline $950 / 970 / 1014$ & Phosphate stretching & DNA \\
1238 & C-O stretching & Collagen I \\
1294 & Deformation N-H cytosine & $?$ \\
1390 & Amide III & Collagen/proteins \\
1658 & Stretching $\mathrm{C}=\mathrm{O} /$ Amida I & Collagen \\
$1676-89$ & Stretching C=O/Amida I/ $\beta$-sheet & proteins \\
$1729 / 1770$ & Stretching C=O & Lipids \\
2958 & $\mathrm{v} \mathrm{CH}$ & Lipids membrane \\
2958 & $\mathrm{v} \mathrm{CH} / \mathrm{CH}_{3}$ & Phospholipids \\
2973 & $\mathrm{v}$ ass $\mathrm{CH}_{3}$ & Lipids/fatty acids \\
$3251-3529$ & Stretching ass O-H & Confined water \\
\hline
\end{tabular}

\section{Materials and methods}

This research was carried out in accordance to the ethical principles and it was approved by the local ethical research committee (protocol 032/2010 PH-CEP). Patients were informed about the subject of the research and signed the informed consent.

\subsection{Sample procedures}

Samples were obtained from biopsies of 14 patients with AC diagnosis and 14 NM (normal). NM samples were obtained from lower lip biopsies when an extension to healthy tissues was needed in order to complete the surgical procedure. Fragments from each tissue sample were identified, snap frozen and stored in liquid nitrogen $(-80 \mathrm{C})$ until FT-IR spectra were recorded.

\subsection{FT-IR microspectroscopy}

A Varian INC 660/610 FT-IR spectrometer was used at $4 \mathrm{~cm}^{-1}$ resolution. A background was made with 800 scans, and each spectrum was recorded with 400 scans. FT-IR background removal was performed by the collection of a spectrum of the air before analysis. Then equipment performed background removal automatically while spectra were collected. FT-IR spectra were measured at five different points across each sample resulting in 70 AC spectra and $70 \mathrm{NM}$ spectra.

The thawing of the samples was performed using saline $(0.9 \%$ $\mathrm{NaCl}$ Aster $\left.^{\circledR}\right)$. After thawing, clinical samples were placed on a platinum substrate with epithelium faced up, following FT-IR measurements. To mimic an in vivo layout, beam of light first passed through the epithelium and then the connective tissue. Spectra were then obtained with micro FT-IR spectrometer with the aid of accessory attenuated total reflection (ATR-Attenuated Total Reflection). Thus, the spectra were obtained when there was a slight contact between the sample and the ATR crystal accessory. 


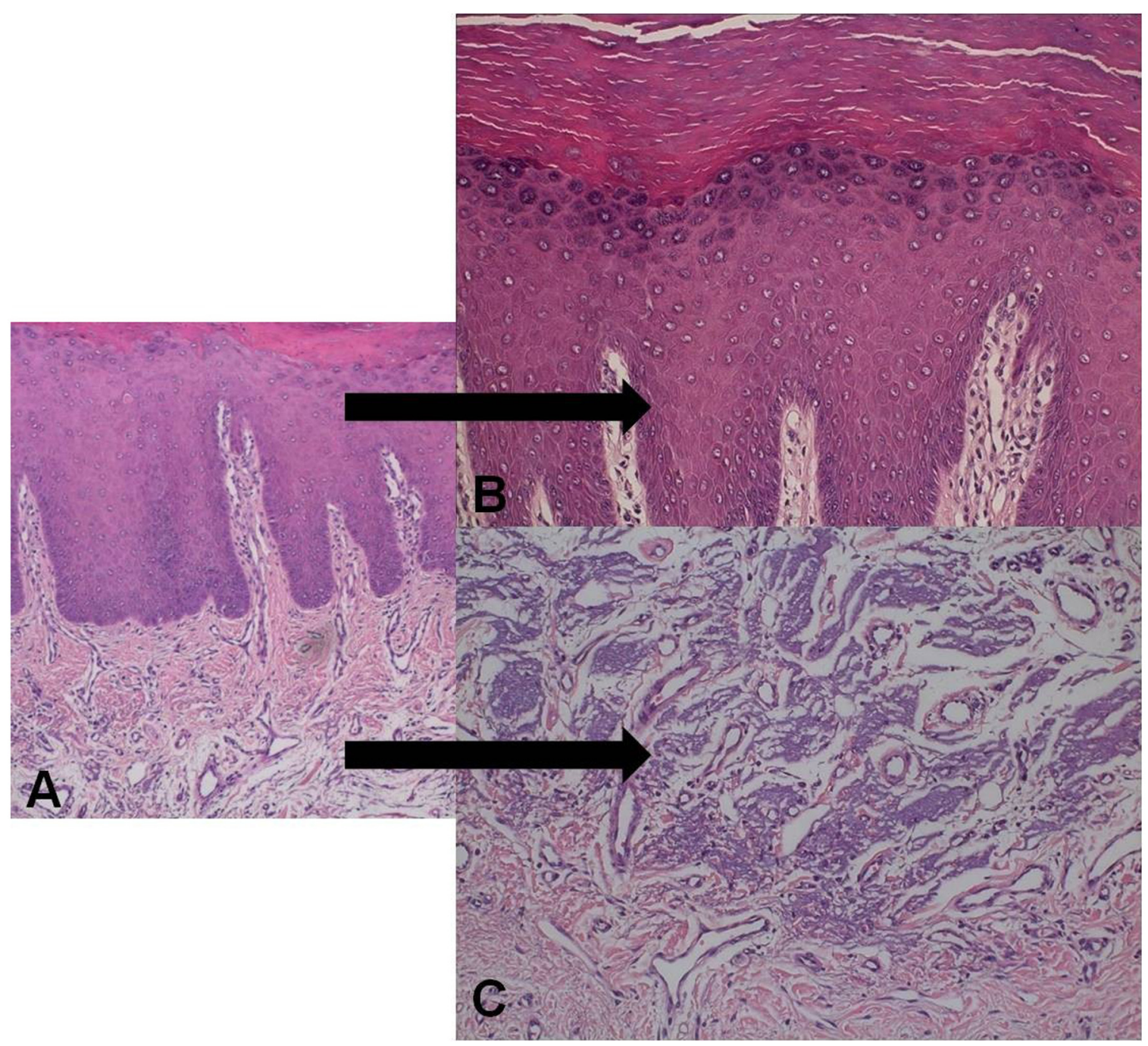

Fig. 2. A) AC histopathological samples (HE-10x), showing in; B) the characteristics of the epithelium as severe acanthosis, and a thick layer of keratin (HE-40x), and, C) detail of basophilic degeneration collagen, which was evident in all samples of AC (HE, 40x).
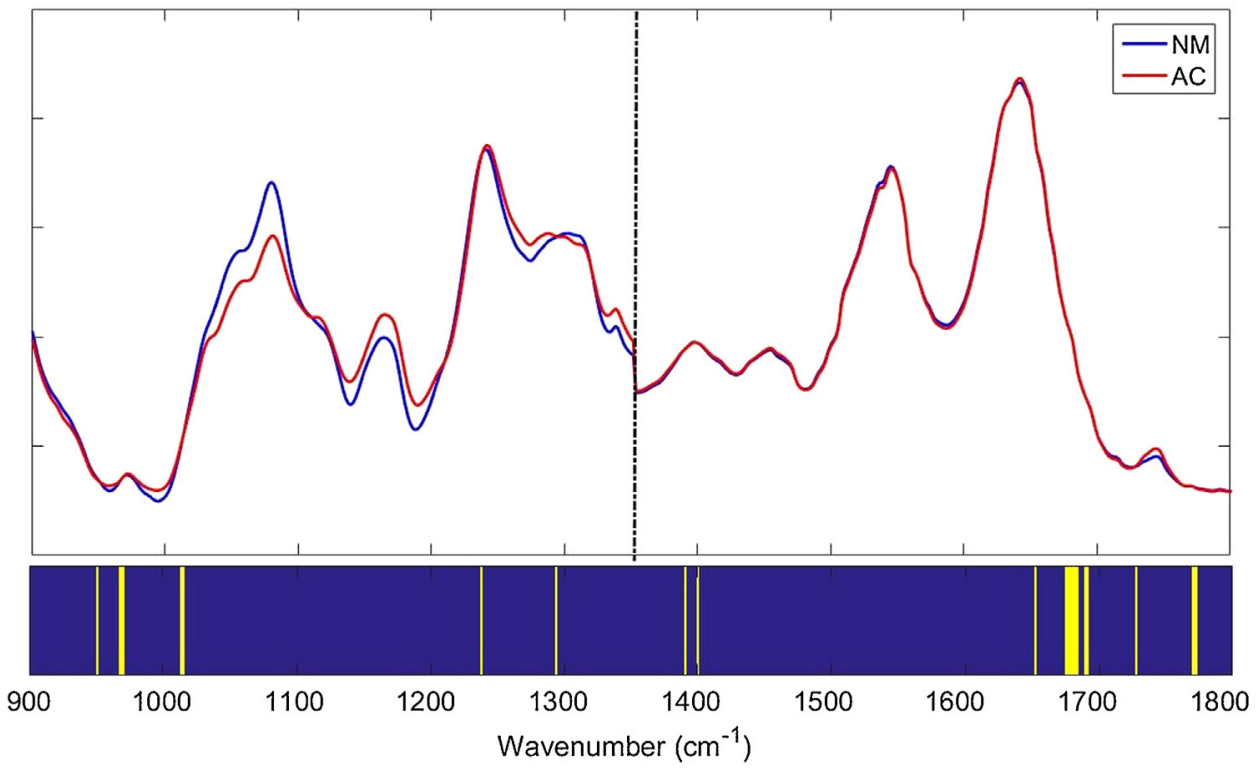

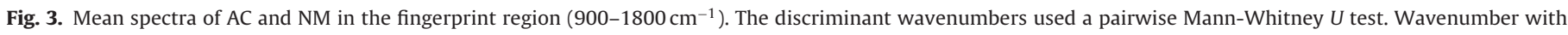

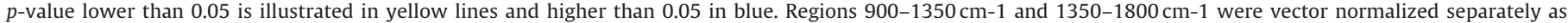
indicated by dotted black line (for interpretation of the references to colour in this figure legend, the reader is referred to the web version of this article). 


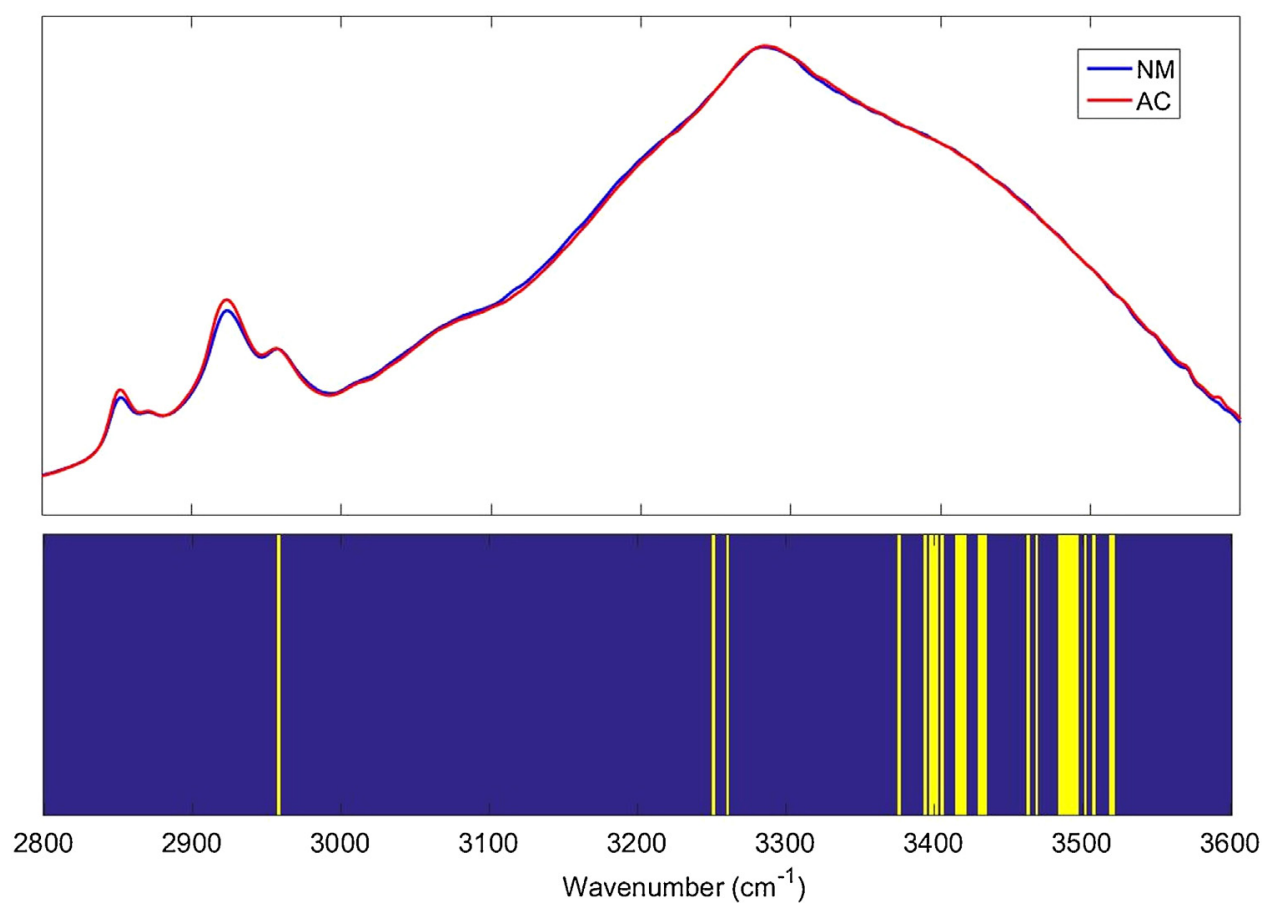

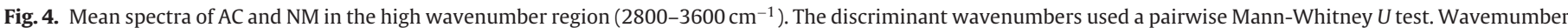

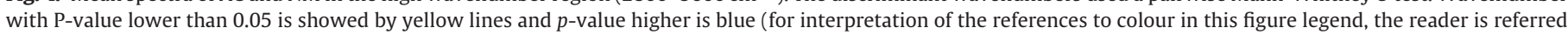
to the web version of this article).

\subsection{Histopathology}

All samples were fixed in $10 \%$ formaldehyde solution for further histopathologic tissue analysis at the Oral Pathology Laboratory, Department of Biosciences and Oral Diagnosis, Institute of Science and Technology, UNESP- Univ. Estadual Paulista.

The stain used was hematoxylin and eosin (H\&E), following the staining protocol steps: first, the sections were deparaffinized with xylene for $10 \mathrm{~min}$, followed by re-hydration with absolute alcohol for 5 min, and washed with 95\% alcohol for 2 min and then $70 \%$ alcohol for $2 \mathrm{~min}$. After a quick wash in distilled water, the slides were taken into solution with hematoxylin and then counterstained with eosin.

\subsection{Data analysis}

\subsubsection{Mann-Whitney U Test}

The spectra dataset were imported into the software written in-house in the MATLAB environment. Data pre-processing included the restriction of the wavenumber range between 900 and $1800 \mathrm{~cm}^{-1}$ in the fingerprint region also between 2800 and $3600 \mathrm{~cm}^{-1}$ in the high wavenumber region. All spectra were vector normalized in the follow regions: $900-1350 \mathrm{~cm}^{-1}$, $1350-1800 \mathrm{~cm}^{-1}$ and $2800-3600 \mathrm{~cm}^{-1}$. In order to verify the statistical difference in the spectra, Mann-Whitney $U$ test was performed in each variable (wavenumber) with $p$-value $<0.05$.

In order to verify the discriminant IR spectral wavenumbers between the normal and actinic cheilitis, a pairwise Mann-Whitney $U$ test were performed with the intensity values. The results from this analysis are shown using the concept of spectral barcodes with $0.05 p$-value thresholds.

\subsubsection{Principal component analysis/Linear discriminant analysis}

For multivariate analysis, all spectra were split into two distinct matrices that contained respectively the fingerprint regions and high wavenumber regions. The principal component analysis (PCA), followed by linear discriminant analysis (LDA) with 5-fold cross validation, was performed in each dataset separately for spectra classification.

The ROC curve (Recevier Operating Characteristic) was calculated to analyze the performance of the classification. To check the sensitivity and specificity of the study, the area under the ROC curve was calculated, showing an association of the discriminating power of a diagnostic model.

\section{Results}

\subsection{Histopathology}

Histological analysis of NM samples showed mucosal lining with appearance of normality and lamina propria with collagen fibers arranged in parallel bundles and cellular characteristic component. Samples of AC group revealed acanthotic epithelium with epithelial atypia ranging from mild and moderate, and hyperkeratinization. The connective tissue showed solar elastosis, basophilic degeneration of collagen fibers and vessels scattered throughout the stroma. Inflammatory cells were also observed (Fig. 2).

\subsection{FT-IR spectra analysis}

The Figs. 3 and 4 shows the mean spectra of NM and AC. Visually it was difficult to discriminate between the samples in the figure. (Fig. 3). After the Mann-Whitney $U$ test the vibrational modes of $\mathrm{CO}$ (Collagen 1 ), $\mathrm{PO}_{2}$ (Nucleic Acids) and $\mathrm{C}=\mathrm{O}$ asymmetric (Triglycerides/Lipids) were observed as a possible spectral biomarker. These bands were chosen because they represent the vibrational modes related to collagen and DNA, which are supposedly related to changes in AC samples.

Analyzing the barcodes related to the spectra, it was possible to make a correlation between the groups using their principal features concerned to the specific vibrational mode and structural component (Figs. 3 and 4). 


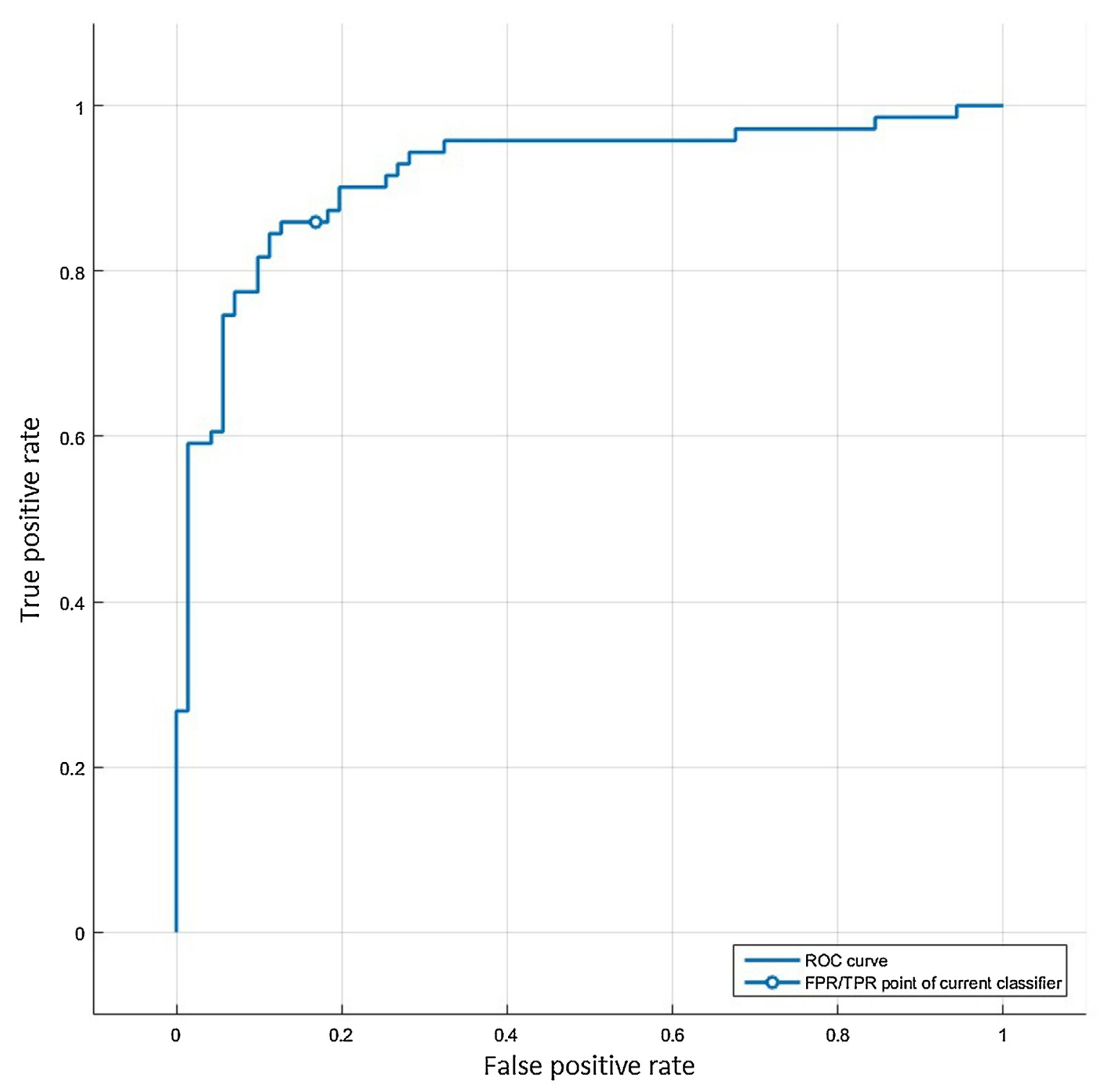

Fig. 5. ROC curve of fingerprint region $\left(900-1800 \mathrm{~cm}^{-1}\right)$.

PCA/LDA was performed in fingerprint and high wavenumber regions separately. For the fingerprint regions the first 51 PCs that represent more than $99 \%$ of all variance in the dataset were used; for the high wavenumber regions 40 were used.

In the fingerprint region, the bands which showed a higher spectral difference were mainly related to the $\mathrm{C}-\mathrm{C}$ and $\mathrm{CH}$ vibrations corresponding to collagen and nucleic acids DNA and RNA. High wavenumber region was more related to lipid structures and confined water molecules variations in the tissues (Table 1 ). The yellow bars in Figs. 3 and 4 are related to the spectral regions with had a statistical relevant result comparing both groups. Specificity was $83 \%$ for fingerprint region and $74 \%$ for high wavenumber. Sensibility was $85,9 \%$ for fingerprint region and $80,2 \%$ for high wavenumber.

A diagnostic model was performed using the results of PCA/LDA analysis. ROC curve was used to evaluate the specificity and sensitivity of the diagnostic model. The value of the predictive model corresponding to the area under the curve was 0.91 for the fingerprint region and 0.83 for the highwavenumber region (Figs. 5 and 6 ), showing the greater accuracy of the test.

\section{Discussion}

AC is a potentially malignant disorder $[3-6,10,11]$ and it was possible to observe changes in nucleic acid both in the spectra analysis and in the results of Mann-Whitney test. The cell cycle may have an effect on these results, as nucleic acids are directly involved in the process of synthesis and cell multiplication; it also increases the production of collagen fibers. In the present study, the major changes from the spectra included mainly collagen degeneration, caused by UV radiation and variations in nucleic acids (DNA and RNA), which may also be associated with radiation.

The pathogenesis and the development of cancer is a multifactorial process controlled by several genes. The activation of oncogenes and inactivation of tumor suppressors in the carcinogenesis process causes significant changes in the structure of proteins, nucleic acids and intracellular lipids. In order to expose the above changes, FTIR spectroscopy can add to other methods of analysis enabling the diagnosis of neoplastic cells at the molecular level [12-15]. However it is not possible to determine from spectroscopy techniques exactly which oncogenes or tumor suppressors are altered. 


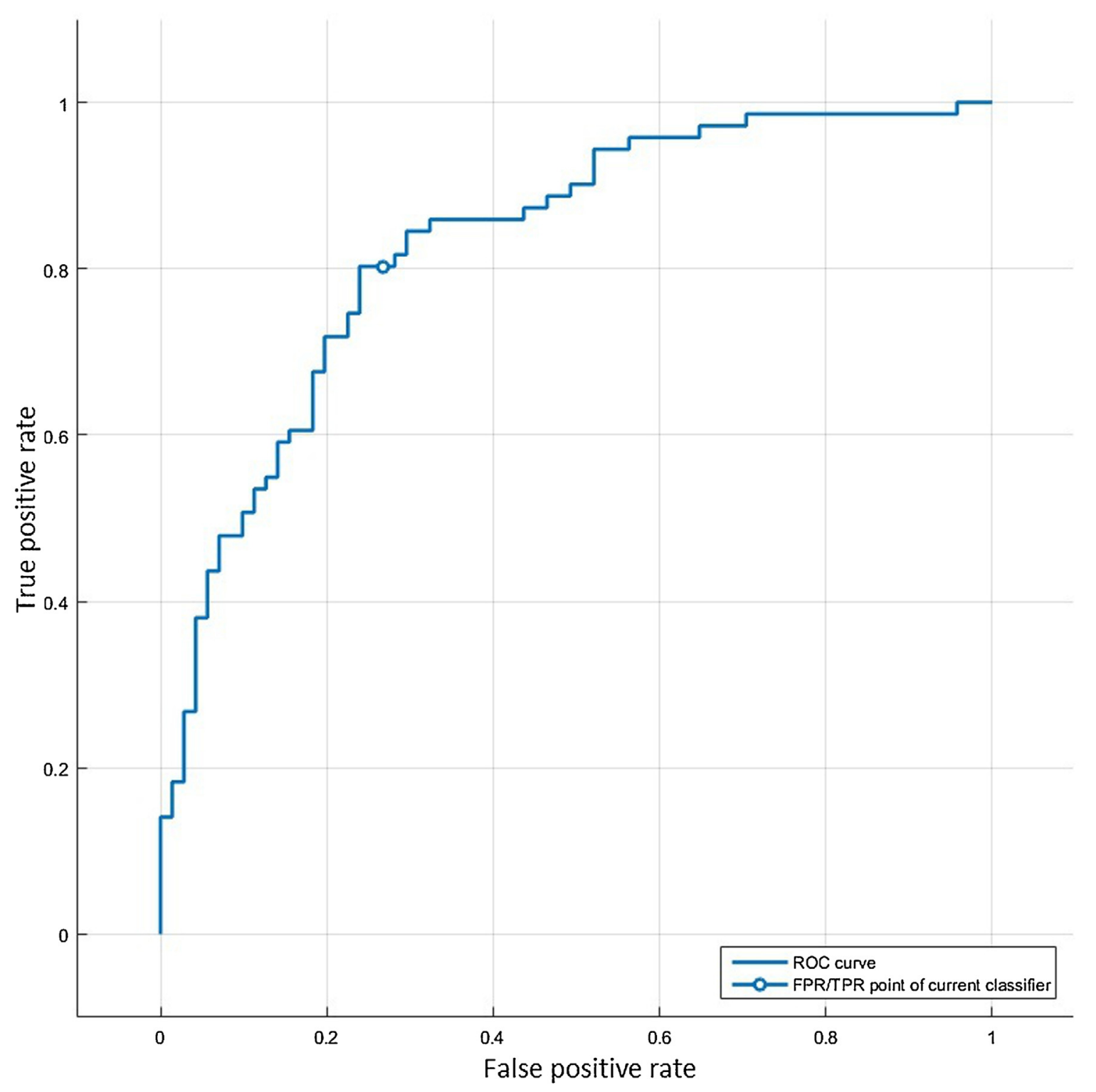

Fig. 6. ROC curve of high wavenumber region $\left(2800-3600 \mathrm{~cm}^{-1}\right)$.

DNA double strand breaks (DSBs) are considered one of the most hazardous lesions due to the fact that they may result in DNA fragment loss or in chromosome rearrangements. Of special interest, ring and dicentric chromosomes may initiate breakage-fusionbridge cycles that promote the main genomic alterations observed in cancer cells [16-18]. Besides this, DNA damage is inflicted, causing cell activation and a hierarchical cascade of proteins composed of sensors, transducers and effectors, widely known as the DNA damage response [18].

Fourtanier et al., 2011 [19] stated that short and long-term adverse effects are induced to skin by both acute and repeated sun exposure. The same occurs to lips. They also explain that the high intensity of artificial UV sources penetrate deeper into the skin causing a wide variety of biological damage. UV radiation produces reactive oxygen species (ROS) mainly through the interaction with endogenous and exogenous chromophores. ROS cause damage to DNA, cells, vessels and tissues [19]. ANOVA test allowed analyzing the damage as DNA and collagen spectral regions had greater peaks variations.

In vitro reconstructed human skin, composed of a living dermal equivalent and a fully differentiated epidermis, exposed to
UV induced the expression of numerous genes in fibroblasts and keratinocytes. These genes are involved in extracellular matrix homeostasis, oxidative stress, cell growth, inflammation and epidermal differentiation [20].

Basophilic degeneration of collagen that occurs in the underlying connective tissue, mainly caused by the effect of UV radiation, is a histopathological indication for the diagnosis of AC.

Although cell cycle control markers, as p53, were not assessed, it is well-known changes in this control process are closely related to malignancy. Ranganathan \& Kavitha, (2012) [21] reported an association between $\mathrm{p} 53$ and consequent inhibition of DNA replication, suggesting a strong association of gene expression carcinogenesis process in the oral epithelium. Although we did not point the altered markers in the cell cycle [20], it was possible to demonstrate the association of altered DNA band peak in AC samples.

Another important detail concerning FT-IR technique and its potential as a tool for diagnostic pathology is the reduction of time and the length of the sample. The time required for spectra acquisition is approximately six minutes as quoted in the present study, and according to other authors [13,23]. After obtaining the spectrum, it takes approximately $10 \mathrm{~min}$ for data treatment (baseline 


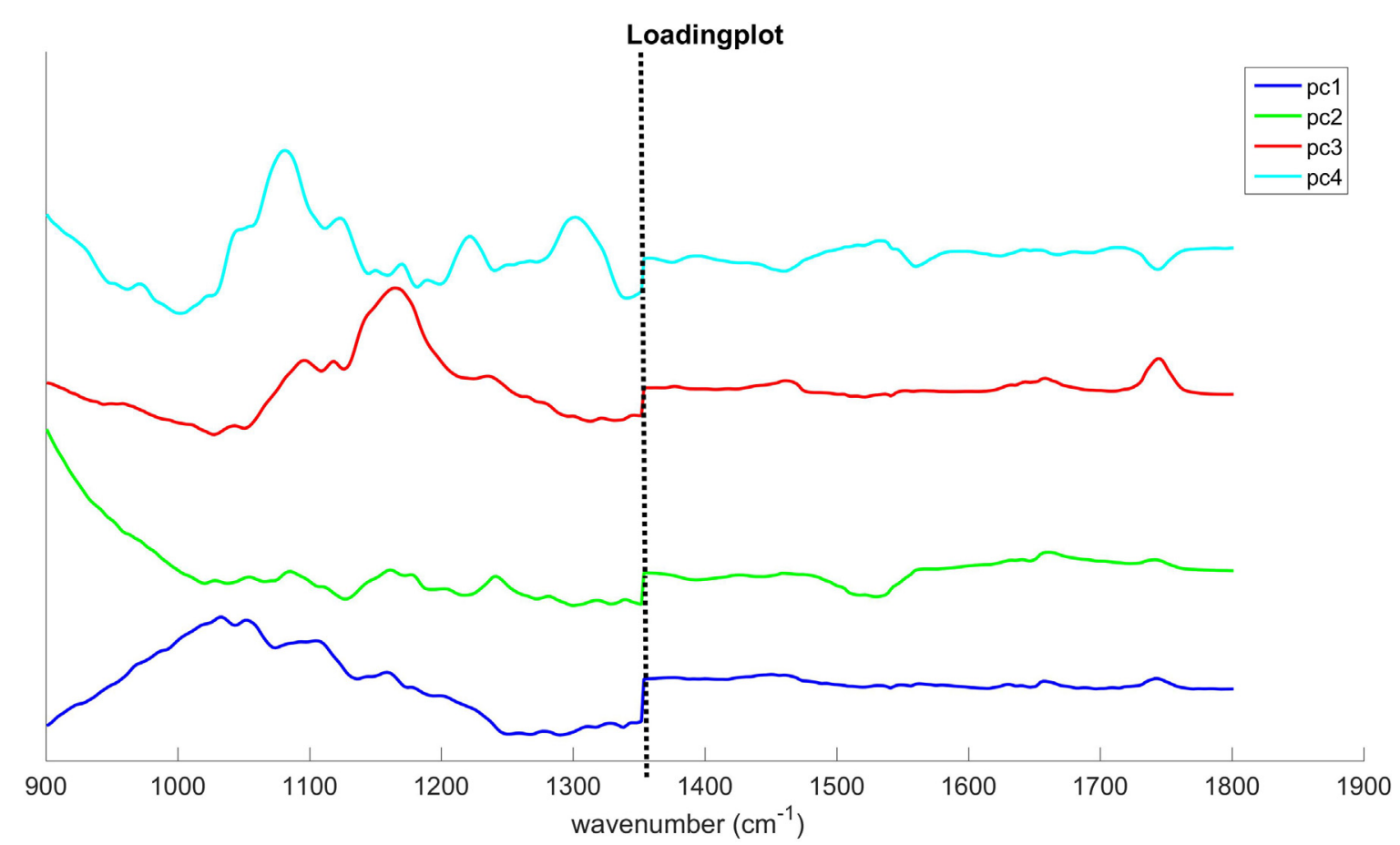

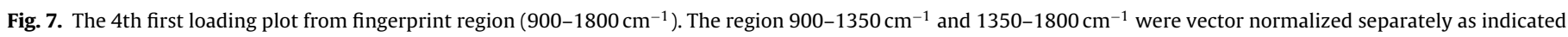
by dotted black line.

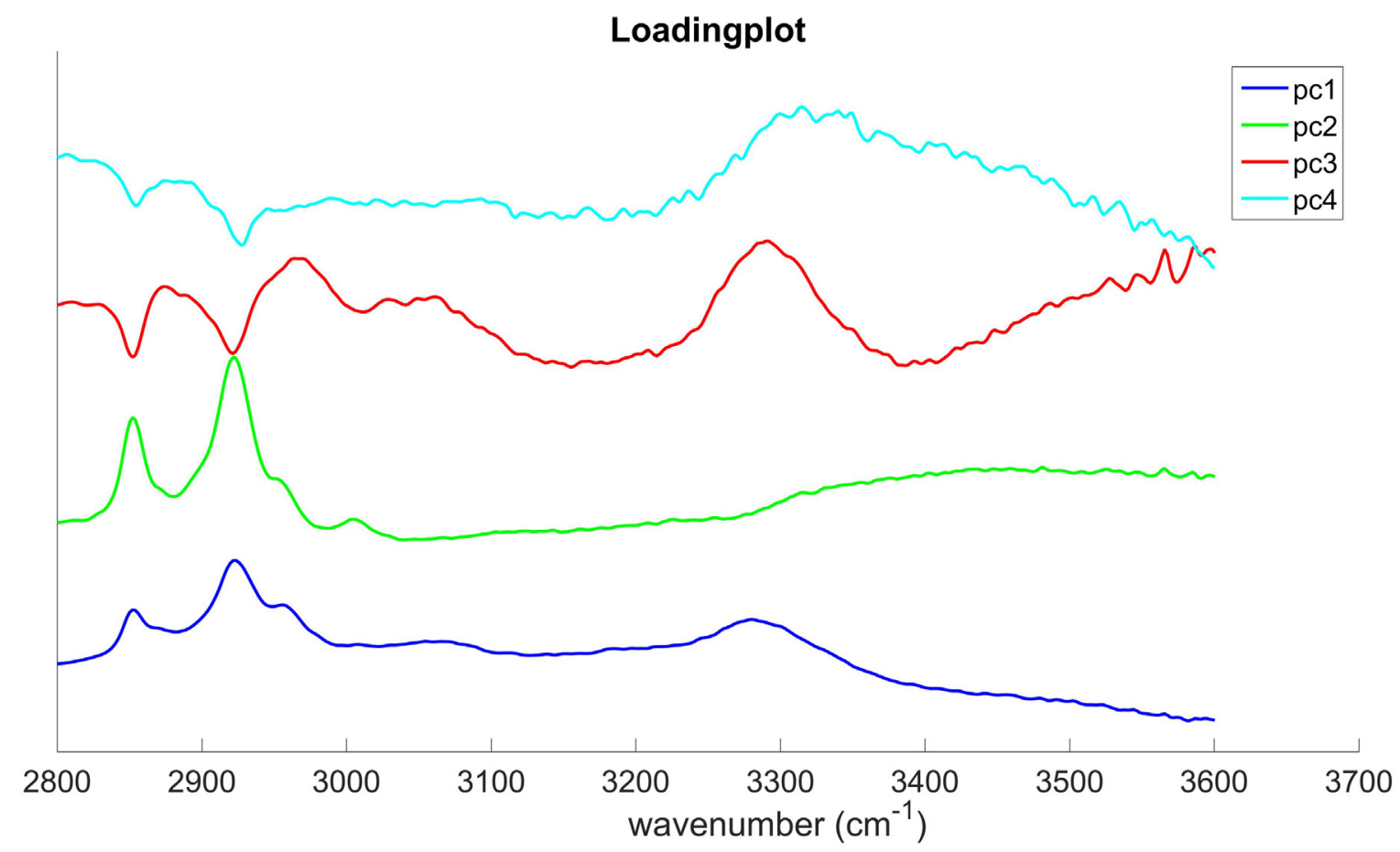

Fig. 8. The 4 th first loading plot from high wavenumber region.

subtraction, normalization, statistics analysis). Thus, 15-20 min are required for molecular analysis of the spectra, much faster than processing tissue for microscopic examination. Spectroscopic analysis sample may be smaller than $1 \mathrm{~cm}$ [3], different from the necessary for histopathological analysis.

Li et al. (2005) studied gastric cancer using FT-IR spectroscopy and showed that spectral features were related to the changes in structure and composition of biological molecules such as proteins, nucleic acids, and fats. Their results indicate that FT-IR spectroscopy combined with appropriate pattern recognition algorithms, can distinguish benign from malignant disease [24]. Indeed, the present study showed that it is possible to discriminate potentially malignant disorders from NM using suitable methods for data analysis like PCA and LDA(Figs. 7 and 8).

Many authors have reported the importance of the optical biopsy techniques (Raman, FT-IR, Fluorescence, UV-vis, and others) mainly due to the possibility of obtaining a concrete diagnosis $[12,14,15,25,26]$. In spite of this, it is also important to consider that 
the vibrational spectroscopy (FT-IR and Raman) shows relevant information for understanding the behavior of macromolecules in physiological and pathological states that cannot be studied by morphological analysis.

Considering the limitations of this study, the results allowed us to consider that micro FT-IR spectroscopy permits evaluation of the spectral region of nucleic acids, showing a more active and intense cell cycle in AC tissue when compared to NM tissue which may lead to malignant transformation into SCC. Also we observed other variations troughs PCA analysis such as in collagen, lipids and confined water.

\section{Conclusions}

FT-IR analysis of AC may demonstrate that spectral regions of collagen and nucleic acids are statistically significant when compared to the NM samples. The technique associated with PCA/LDA can still provide additional information regarding the vibrational modes of proteins, lipids and confined water when evaluating the regions of fingerprint and highwavenumber.

\section{Conflict of interest}

The authors declare no conflict of interest and also that the paper has not been published elsewhere.

\section{Acknowledgments}

The authors would like to thank the Brazilian agencies FAPESP (Process 2012/50935-8 and 2011/06615-8), CAPES and CNPq for financial support; the Laboratory of Oral Pathology from the Department of Biosciences and Oral Diagnosis, Institute of Science and Technology, Univ Estadual Paulista - UNESP for histophatological analysis. Authors are also grateful to Multiusers Central Facilities of UFABC for the experimental support.

\section{References}

[1] L.F.C.S. Carvalho, R.A. Bitar, E.A.L. Arisawa, A.A.H. Brandão, K.M. Honório, L.A.G. Cabral, et al., Spectral region optimitazion for Raman-based optical biopsy of inflammatory lesions, Photomed. Laser Surg. 28 (2010) 111-117.

[2] S.D. da Silva, A. Ferlito, R.P. Takes, R.H. Brakenhoff, M.D. Valentin, J.A. Woolgar, et al., Advances and applications of oral cancer basic research, Oral Oncol. 47 (2011) 783-791.

[3] A.S. Cavalcanti, A.L. Anbinder, Y.R. Carvalho, Actinic cheilitis: clinical and histological features, J. Oral Maxillofac. Surg. 66 (2008) 498-503.

[4] M.M.S. Nico, E.A. Rivitti, S.V. Lourenço, Actinic cheilitis: histologic study of the entire vermilion and comparison with previous biopsy, J. Cutan. Pathol. 34 (2007) 309-314.
[5] I. Van der Waal, Potentially malignant disorders of the oral and oropharyngeal mucosa; present concepts of management, Oral Oncol. 45 (2009) 317-323.

[6] F.C. Sgarbi, F. Bertini, T.M. Tera, A.R. Cavalcante, Morphology of collagen fibers and elastic system fibers in actinic cheilitis, Indian J. Dent. Res. 21 (2010) $518-522$.

[7] F.M. Lyng, E.Ó. Faoláin, J. Conroy, A.D. Meade, P. Knief, B. Duffy, et al., Vibrational spectroscopy for cervical cancer pathology, from biochemical analysis to diagnostic tool, Exp. Mol. Pathol. 82 (2007) 121-129.

[8] L.F.C.S. Carvalho, E.T. Sato, J.D. Almeida, H.S. Martinho, Diagnosis of inflammatory lesions by high-wavenumber FT-Raman spectroscopy, Theor. Chem. Acc. 130 (2011) 1221-1229.

[9] S.C.G. Penteado, B.P. Fogazza, C.S. Carvalho, E.A.L. Arisawa, M.A. Martins, A.A. Martin, et al., Diagnosis of degenaritive lesions of supraspinatus rotator cuff tendons by Fourier transform-Raman spectroscopy, J. Biomed. Opt. 13 (2008) $1-10$.

[10] G. Thomas, M. Hashibe, B.J. Jacob, Risk factors for multiple oral premalignant lesions, Int. J. Cancer 107 (2003) 285-291.

[11] M. Ulrich, S. González, B. Asschenfeldt-Lange, J. Roewert-Huber, W. Sterry, E. Stockfleth, et al., Noninvasive diagnosis and monitoring of actinic cheilitis with reflectance confocal microscopy, J. Eur. Academ. Dermatol. Venearol. 25 (2011) 276-284.

[12] C. Conti, P. Ferraris, E. Giorgini, C. Rubini, S. Sabbatini, G. Tosi, et al., Microimaging FTIR of head and neck tumours, Microsc. Res. Tech. 72 (2009) 67-75.

[13] C. Petibois, G. Deléris, Chemical mapping of tumor progression by FT-IR imaging: towards molecular histopathology, Trends Biotechnol. 24 (2006) 455-462.

[14] J. Wang, J. Shi, Y. Xu, X. Daun, L. Zhang, J. Wang, et al., FT-IR spectroscopic analysis of normal and cancerous tissues of esophageous, World J. Gastroenterol. 9 (2003) 1897-1899.

[15] S. Lin, M. Li, W. Cheng, FT-IR and Raman vibrational microspectroscopies used for spectral biodiagnosis of human tissues, Spectroscopy 21 (2007) 1-30.

[16] S.E. Artandi, R.A. DePinho, A critical role for telomeres in suppressing and facilitating carcinogenesis, Curr. Opin. Genet. Dev. 10 (2000) 39-46.

[17] D. Gisselsson, J. Bjork, M. Hoglund, F. Mertens, P. Dal Cin, M. Akerman, et al., Abnormal nuclear shape in solid tumors reflects mitotic instability, Am. J. Pathol. 158 (2001) 199-206.

[18] M. Terradas, M. Martin, L. Hernández, L. Tusell, A. Genescà, Nuclear envelope defects impede a proper response to micronuclear DNA lesions, Mutat. Res. 729 (2012) 35-40

[19] A. Fourtanier, D. Moyal, S. Seite, UVA filters in sun protection products, Photochem. Photobiol. Sci. 11 (2012) 81-89.

[20] C. Duval, R. Schimdt, M. Regnier, V. Facy, D. Asselineau, F. Bernerd, The use of reconstructed human skin to evaluate UV-induced modifications and sunscreen efficacy, Exp. Dermatol. 12 (2003) 64-70.

[21] K. Ranganathan, R. Kavitha, Proliferation and apoptosis markers in oral submucous fibrosis, J. Oral Maxillofac. Pathol. 15 (2012) 148-153.

[23] J.C. Miller, J.H. Thrall, Clinical molecular imaging, J. Am. Coll. Radiol. 1 (2004) 4-23.

[24] Q. Li, X. Sun, Y. Xu, L. Yang, Y. Zhang, S. Weng, et al., Use of Fourier-transform infrared spectroscopy for rapidly diagnose gastric endoscopic biopsies, World J. Gastroenterol. 11 (2005) 3842-3845.

[25] C. Kendall, J. Day, J. Hutchings, B. Smith, N. Sheperd, H. Barr, et al., Evaluation of Raman probe for oesophageal cancer diagnostics, Analyst 135 (2010) 3038-3041.

[26] R. Malini, K. Venkatakrishna, J. Kurien, K.M. Pai, L. Rao, V.B. Kartha, et al., Discrimination of normal, inflammatory, premalignant, and malignant oral tissue: a Raman spectroscopy study, Biopolymers 81 (2006) 179-193.

[27] Z. Movasaghi, S. Rehman, I.U. Rehman, Fourier transform infrared (FTIR) spectroscopy of biological tissues, Appl. Spectrosc. Rev. 43 (2) (2008) 134-179. 